\title{
Impact of dialysis modality on long-term outcomes in kidney transplantation recipients: a propensity-matched cohort study
}

\author{
Jin Hyuk Paek, Ohyun Kwon, Yaerim Kim, Woo Yeong Park, Kyubok Jin, Seungyeup Han
}

Division of Nephrology, Department of Internal Medicine, Keimyung University Dongsan Medical Center, Daegu, Korea

Background: Kidney transplantation (KT) is the ideal therapy for patients with end stage renal disease. However, pre-emptive KT is not always possible, most patients undergo peritoneal dialysis (PD) or hemodialysis (HD) while awaiting KT. Previous studies analyzing the impact of pre-transplant dialysis modality on patient and graft survival were conflicting and follow-up period is not sufficient. We evaluated the relationship of pre-transplant dialysis modality with long-term clinical outcomes by using propensity score matching method.

Methods: We conducted a retrospective cohort study of 590 patients who underwent KT at Keimyung University Dongsan Medical Center from 2003 to 2016. Of the 590 KT recipients, we excluded pre-emptive KT, second or third KT and 470 recipients were analyzed.

Results: Among $470 \mathrm{KT}$ recipients, 95 recipients (20.2\%) were treated with PD before KT. After using propensity score matching method, 93 recipients were included in each group. The mean follow-up duration was $98.6 \pm 47.3$ months. In the entire cohort, PD group had lower creatinine level at 3 years after KT than HD group $(P=0.030)$. However, there was no significant differences between two groups after matching $(P=0.055)$. Delayed graft function $(P=0.662)$ and biopsy proven acute rejection within 1 year after KT $(P=0.445)$ were comparable between the groups. Ten-year patient survival rates $(P=0.521)$ and 10 -year death-censored graft survival rates $(P=0.407)$ were similar between two group. In Cox proportional hazard model, pre-transplant dialysis modality was not an independent risk factor for the patient mortality, graft failure, and death-censored graft failure.

Conclusions: Pre-transplant dialysis modality of PD or HD did not influence on the long-term patient and graft survival after KT. Moreover, short-term complications were similar between two groups.

Corresponding author: Jin Hyuk Paek

E-mail: novawang@naver.com

(c) The Korean Society for Transplantation

This is an Open Access article distributed under the terms of the Creative Commons Attribution Non-Commercial License (http://creativecommons.org/licenses/by-nc/4.0/) which permits unrestricted non-commercial use, distribution, and reproduction in any medium, provided the original work is properly cited. 